\title{
Influence du remplacement d'une partie du suif d'un aliment d'allaitement par de la tricaproïne ou de la tricapryline en association à de l'huile de coprah sur la croissance du jeune veau préruminant
}

\author{
B. AUROUSSEAU, P. THIVEND * et M. VERMOREL \\ Avec la collaboration technique de C. Marpillat*, Mariette Martinaud \\ et Françoise DuboISSET \\ I.N.R.A., Laboratoire d'Etude du Métabolisme énergétique \\ * Laboratoire de la Digestion \\ C.R.Z.V. Theix, F 63122 Ceyrat
}

\section{Résumé}

72 veaux séparés en 3 lots ont été utilisés pour comparer l'efficacité d'utilisation pour la croissance de 3 laits de remplacement comportant 23,4 p. 100 de matières azotées et 21 p. 100 de lipides : un lait «témoins où les matières grasses étaient apportées par du suif (lot 1) et 2 laits expérimentaux dans lesquels $2 / 3$ du suif étaient remplacés en parties égales par de l'huile de coprah et par de la tricaproïne (lot 2 ) ou par de l'huile de coprah et par de la tricapryline (lot 3).

L'état sanitaire des veaux était médiocre et 5 d'entre eux sont morts pendant la première semaine d'essai portant à 21,24 et 22 veaux les effectifs respectifs des 3 lots d'animaux. La fréquence des diarrhées ou de vitesses de croissance et d'efficacité alimentaire anormalement faibles ont été plus importantes dans le premier lot.

Les veaux des lots 2 et 3 ont présenté des vitesses de croissance $(+40$ p. 100) et une efficacité alimentaire $(+26$ p. 100) supérieures à celles observées chez les veaux du lot 1 au cours des 3 premières semaines d'expérience. L'accroissement de l'effícacité d'utilisation de l'énergie pour la croissance a été encore plus important : $+30 \mathrm{p} .100$ après 3 semaines. Il demeure significatif après 5 semaines : +17 p. $100(P<0,05)$.

Après élimination des données obtenues chez les veaux présentant des vitesses de croissance particulièrement lentes afin de supprimer les effets liés à l'état sanitaire des animaux, l'efficacité alimentaire demeure plus élevée $(+14$ p. $100, \mathrm{P}<0,05)$ chez les veaux des lots 2 et 3 lors des 3 premières semaines d'expérience. Les 2 types d'effets de l'ingestion d'acides gras à chaîne moyenne : amélioration de l'état sanitaire des veaux ou effet direct sur l'efficacité de fixation de tissus, sont discutés.

Mots clés : veau préruminant, acide caprylique, acide caprö̈que, efficacité alimentaire.

\section{Introduction}

Les matières grasses du lait (RAven \& Robinson, 1958 ; Raven, 1970), l'huile de coprah (Toullec \& Mathieu, 1969 ; Roy, Stobo \& Gaston, 1970) ou l'huile de palmiste (VEEN, 1970) constituent des sources d'énergie plus intéressantes pour l'ali- 
mentation du jeune veau que les matières grasses comme le suif, le saindoux ou la plupart des autres huiles végétales. L'intérêt de ce type de lipides réside dans leur richesse en acides gras à chaîne moyenne (AGCM). Les triglycérides d'AGCM sont hydrolysés plus efficacement par la lipase salivaire dans la caillette (EDWARDs-WEBB \& Thompson, 1977) ; ils sont également plus sensibles que les triglycérides d'acides gras longs à l'hydrolyse par la lipase pancréatique dans l'intestin (ENTRessangles et al., 1961) et sont facilement et rapidement absorbés aussi bien à travers la paroi de la caillette (EDWARD-WEBB \& THOMPSON, 1978) qu'à travers l'épithélium intestinal (BENNETT, 1964 ; ISSELBACHER, 1968). Ces propriétés leur confèrent une cxcellente digestibilité (Niesar, 1964 ; Toullec \& Mathieu, 1969 ; Veen, 1970). De plus, la fixation de protéines corporelles (VEEN, 1970) et la croissance des animaux (DE Schothorst, 1966 ; Toullec, communication personnelle ; Roy et al., 1973 a) sont également augmentées.

Nous avons envisagé l'emploi d'AGCM purifiés et mis en évidence une augmentation de la fixation de protéines corporelles chez des veaux qui ont reçu des aliments dont les matières grasses étaient apportées en parties égales par du suif, de la tricaproïne ou de la tricapryline et par de l'huile de coprah (Aurousseau, Vermorel \& Bouvier, 1983, 1984). Il nous a paru important de compléter ces premiers résultats par l'étude des effets de ces matières grasses sur la croissance du jeune veau au cours d'une expérience en lot, d'autant plus que chez l'enfant prémature les effets positifs escomptés lorsqu'on introduit des AGCM dans le lait ne sont pas toujours obtenus (Окамото et al., 1982).

\section{Matériel et méthodes}

Trois types d'aliments d'allaitement comportant environ 23,4 p. 100 de matières azotées totales et 21 p. 100 de matières grasses ont été utilisés (tabl. 1) :

- un aliment d'allaitement témoin à base de suif et de poudre de lait écrémé (lait « suif »);

- un aliment d'allaitement expérimental dans lequel $2 / 3$ du suif de l'aliment témoin étaient remplacés en parties égales par de l'huile de coprah et par de la tricaprö̈ne (aliment « suif-coprah-TC6 »);

- un aliment d'allaitement expérimental dans lequel $2 / 3$ du suif de l'aliment témoin étaient remplacés par de l'huile de coprah et par de la tricapryline (aliment " suif-coprah-TC8»).

Les acides gras expérimentaux d'origine industrielle ont été introduits dans les aliments sous forme de triglycérides interestérifiés à du suif et incorporés à la poudre de lait par voie humide. Le lait a ensuite été séché selon le procédé Spray.

Soixante-douze veaux mâles de race Frisonne (2 groupes de 36 animaux) ont été achetés à l'âge de 8 jours et répartis en 3 lots homologues de 24 animaux après une semaine d'observation au cours de laquelle ils ont reçu l'aliment témoin. A partir du quinzième jour, chaque lot a reçu pendant 5 semaines l'un des 3 aliments décrits ci-dessus. 
TABLEAU 1

Composition des aliments d'allaitement $(\mathrm{g} / \mathrm{kg}$ poudre de lait).

Milk composition ( $\mathrm{g} / \mathrm{kg}$ milk powder).

\begin{tabular}{|c|c|c|c|}
\hline $\begin{array}{l}\text { Nature du lait } \\
\text { Milk type }\end{array}$ & $\begin{array}{l}\text { «Suif » } \\
\text { «Tallow» } \\
\text { milk replacer }\end{array}$ & $\begin{array}{c}\text { « Suif-coprah-TC6 } \\
\text { « Tallow-coconut } \\
\text { oil-TC6 } \\
\text { milk replacer }\end{array}$ & $\begin{array}{l}\text { « Suif-coprah-TC8 } \\
\text { «Tallow-coconut } \\
\text { oil-TC8 } \\
\text { milk replacer }\end{array}$ \\
\hline $\begin{array}{l}\text { Suif } \ldots \ldots \cdots \cdots \cdots \\
\text { Tallow }\end{array}$ & 215 & $71^{* 6}$ & $71^{*}$ \\
\hline $\begin{array}{l}\text { Tricaproïne } \ldots \ldots \ldots \ldots \ldots \\
\text { Tricaproin }\end{array}$ & - & $72 *$ & - \\
\hline $\begin{array}{l}\text { Tricapryline } \\
\text { Tricaprylin }\end{array}$ & 一 & - & $72 *$ \\
\hline $\begin{array}{l}\text { Huile de coprah } \ldots \ldots \ldots \ldots \\
\text { Cocontut oil }\end{array}$ & - & 72 & 72 \\
\hline $\begin{array}{l}\text { Poudre de lait écrémé } \ldots . . . \\
\text { Skim-milk powder }\end{array}$ & 655 & 655 & 655 \\
\hline $\begin{array}{l}\text { Poudre de lactosérum } \ldots \ldots \\
\text { Whey powder }\end{array}$ & 84 & 84 & 84 \\
\hline $\begin{array}{l}\text { Emuisifiart } \\
\text { Emulsifier }\end{array}$ & 25 & 25 & 25 \\
\hline $\begin{array}{l}\text { Complément minéral et vitami- } \\
\text { nisé } \ldots \ldots \ldots \ldots \ldots \ldots \ldots \ldots \ldots \\
\text { Mineral and vitamin comple- } \\
\text { ment }\end{array}$ & 21 & 21 & 21 \\
\hline $\begin{array}{l}\text { COMPOSITION CHIMIQUE } \\
\text { Chemical composition }\end{array}$ & & & \\
\hline $\begin{array}{l}\text { Matière sèche }(\%) \quad \ldots \ldots \ldots \ldots \\
\text { Dry matter }(\%)\end{array}$ & 95,4 & 96,5 & 95,9 \\
\hline $\begin{array}{lll}\text { Energie } & (\mathrm{MJ} / \mathrm{kg} & \mathrm{MS}) \\
\text { Energy } & (M J / \mathrm{kg} & \mathrm{DM})\end{array}$ & 21,69 & 20,69 & 20,69 \\
\hline $\begin{array}{l}\text { Protéines }(\mathrm{g} / \mathrm{kg} \text { MS }) \ldots \ldots \\
\text { Protein }(g / \mathrm{kg} D M)\end{array}$ & 234 & 236 & 241 \\
\hline $\begin{array}{l}\text { Lipides }(\mathrm{g} / \mathrm{kg} \text { MS }) \\
\text { Lipids }(g / \mathrm{kg} D M)\end{array}$ & 232 & 233 & 230 \\
\hline
\end{tabular}


TABleau 1 (suite)

\begin{tabular}{|c|c|c|c|}
\hline $\begin{array}{l}\text { Nature du lait } \\
\text { Milk type }\end{array}$ & $\begin{array}{c}\text { «Suif 》 } \\
\text { «Tallow» } \\
\text { milk replacer }\end{array}$ & $\begin{array}{c}\text { «Suif-coprah-TC6» } \\
\text { «Tallow-coconut } \\
\text { oil-TC6 } \\
\text { milk replacer }\end{array}$ & $\begin{array}{l}\text { «Suif-coprah-TC8 } \\
\text { «Tallow-coconut } \\
\text { oil-TC8 } \\
\text { milk replacer }\end{array}$ \\
\hline $\begin{array}{l}\text { NATURE DES ACIDES } \\
\text { GRAS ALIMENTAIRES } \\
(\% \text { AG totaux }) \\
\text { Feed fatty acid composition } \\
(\% \text { total } F A)\end{array}$ & & & \\
\hline $\begin{array}{l}\text { Acide caproïque } \ldots \ldots \ldots \\
\text { Caproic acid }\end{array}$ & - & 35,0 & - \\
\hline $\begin{array}{l}\text { Acide caprylique } \ldots \ldots \ldots \ldots \\
\text { Caprylic acid }\end{array}$ & - & - & 35,0 \\
\hline $\begin{array}{l}\text { Acides laurique et myristique } \\
\text { Lauric and myristic acid }\end{array}$ & 4,2 & 22,8 & 23,0 \\
\hline $\begin{array}{l}\text { Acides palmitique et stéarique } \\
\text { Palmitic and stearic acid }\end{array}$ & 49,6 & 23,1 & 22,8 \\
\hline $\begin{array}{l}\text { Acide oléique } \ldots \ldots \ldots \ldots \ldots \\
\text { Oleic acid }\end{array}$ & 36,8 & 15 & 15,4 \\
\hline $\begin{array}{l}\text { Acide linoléique } \ldots \ldots \ldots \ldots \ldots \\
\text { Linoleic acid }\end{array}$ & 3,5 & 2,0 & 2,1 \\
\hline
\end{tabular}

Les animaux ont été pesés une fois par semaine. Ils ont été alimentés en fonction de leur poids vif selon le plan d'alimentation proposé par Toullec et al. (1978) pour la production de veaux abattus à un poids élevé. Les quantités d'aliment réellement consommées ont été calculées grâce à la pesée individuelle des quantités offertes et éventuellement refusées à chaque repas. L'état sanitaire des animaux a été contrôlé tout au long de l'essai.

La teneur en matière sèche des laits a été déterminée par séchage à l'étuve (48 heures à $80^{\circ} \mathrm{C}$ ), leur teneur en énergie à l'aide d'un calorimètre adiabatique Gallenkamp et leur teneur en azote selon la méthode Kjeldahl. Leur teneur en lipides a été mesurée par pesée après hydrolyse acide et extraction à l'hexanc. La composition en acides gras des lipides a été déterminée, après séparation des esters méthyliques, par chromatographie en phase gazeuse sur colonne capillaire d'acier (100 $\mathrm{m}$ de long, $0,5 \mathrm{~mm}$ de diamètre) garnie de carbowax $20 \mathrm{M}$ traité à l'acide térephtalique. Les conditions opératoires ont été les suivantes : température de l'injecteur et du détecteur : $210^{\circ} \mathrm{C}$, température de la colonne : $190^{\circ} \mathrm{C}$, pression 0,6 bar, utilisation d'un injecteur-diviseur 1/100. 


\section{Résultats}

\section{A. Etat sanitaire}

L'état sanitaire des animaux a été médiocre au début de l'expérience puisque 3 animaux du lot "suif » et 2 animaux du lot "suif-coprah-TC8 " sont morts dès la première semaine tandis que la fréquence des diarrhées a été élevée (tabl. 2). Les diarrhées ont été sensiblement moins sévères (durée inférieure à 3 jours) chez les veaux du lot «suif-coprah-TC6 " et moins fréquentes chez les veaux du lot "suif-coprah-TC8». Cependant, la fréquence des retards de croissance (gains de poids nuls ou négatifs) a été plus élevée dans les lots «suif " $(6$ cas) et "suifcoprah-TC6" ( 5 cas), que dans le lot "suif-coprah-TC8" ( 2 cas) ; enfin 15 cas supplémentaires de gain de poids anormalement faibles (moins de $200 \mathrm{~g} / \mathrm{j}$ ) ont été observés dans le lot «suif» contre 9 cas dans chacun des 2 autres lots (tabl. 2).

\section{B. Vitesse de croissance}

Les veaux décédés au cours de la première semaine n'ont pas été pris en compte pour la comparaison des résultats. Les animaux conservés présentaient en début d'expérience des poids vifs moyens comparables : 47,3 $\pm 0,8,47,8 \pm 0,8$ et $47,8 \pm 0,9 \mathrm{~kg}$ pour les lots "suif ", "suif-coprah-TC6 " et "suif-coprah-TC8 » respectivement.

Lc remplacement de $2 / 3$ de suif de l'aliment témoin par des matières grasses riches en acides gras à chaîne moyenne a été accompagné d'un accroissement de la vitesse de croissance des animaux $(P<0,025)$ dès la deuxième semaine : 42 p. 100 et 49 p. 100 d'augmentation avec les laits "suif-coprah-TC6 " ou « suifcoprah-TC8 » respectivement. Cet effet positif s'est maintenu au cours de la troisième semaine (augmentation de 29 et 23 p. 100 respectivement, $P<0,05$ ) et au cours de la quatrième semaine, mais seulement dans le cas des veaux recevant l'aliment "suif-coprah-TC8 " (augmentation de 17 p. 100, P < 0,05). Au cours de la cinquième semaine, les vitesses de croissance des animaux des 3 lots n'ont pas été significativement différentes (tabl. 3).

Dans ces conditions, les poids des animaux ont été significativement différents $(P<0,05)$ dès la deuxième semaine d'expérience. La différence s'est amplifiée en troisième semaine $(P<0,025)$ et s'est maintenue jusqu'à la fỉn de l'expérience (fig. 1).

\section{Efficacité alimentaire}

L.es qualités moyennes de matière sèche (MS) d'aliment ingérés sont légèrement plus élevées pour les lots "suif-coprah-TC6 " et "suif-coprah-TC8 " que pour le lot "suif" mais les différences ne sont pas significatives (tabl. 3).

En revanche, dès la première semaine d'expérience, l'efficacité alimentaire ( $\mathrm{g}$ gain de poids vif $/ \mathrm{kg}$ MS ingérée) est accrue par l'incorporation d'acides gras à chaîne moyenne ( 29 p. 100 et 18 p. 100 d'augmentation pour les lots « suif-coprahTC6 " ou "suif-coprah-TC8 " respectivement). Cet effet est renforcé en deuxième semaine (34 et 43 p. 100 d'augmentation respectivement) puis diminue à partir de 


\section{TABLEAU 2}

Etat sanitaire des animaux au cours de l'expérience.

Health of the animals during the experiment.

\begin{tabular}{|c|c|c|c|}
\hline $\begin{array}{l}\text { Aliment d'allaitement } \\
\text { Milk replacer }\end{array}$ & $\begin{array}{l}\text { «Suif » } \\
\text { «Tallow» }\end{array}$ & $\begin{array}{c}\text { «uif-coprah-TC6 } \\
\text { «Tallow-coconut } \\
\text { oil-TC6» }\end{array}$ & $\begin{array}{c}\text { «uif-coprah-TC8 } \\
\text { «tallow-cocomut } \\
\text { oil-TC8 }\end{array}$ \\
\hline $\begin{array}{l}\text { Effectif en début d'expérience } \ldots . . \\
\text { Animal number at the beginning }{ }_{j} \\
\text { the experiment }\end{array}$ & 24 & 24 & 24 \\
\hline $\begin{array}{l}\text { Nombre danimaux morts (1) } \ldots \text {. } \\
\text { Mortality }\end{array}$ & 3 & 0 & 2 \\
\hline $\begin{array}{l}\text { Nombre d'arimaux atteints de diar- } \\
\text { rhées } \ldots \ldots \ldots \ldots \ldots \ldots \ldots \ldots \ldots \ldots \ldots \ldots \ldots \\
\text { Number of animals suffering from } \\
\text { diarrhoca }\end{array}$ & 14 & 14 & 10 \\
\hline $\begin{array}{l}\text { Durée moyenne des diarrhées (j) (2). } \\
\text { Mean duration of diarhoca }(d) \text { (2) }\end{array}$ & $3,6(1-10)$ & $2,2(1-3)$ & $3,1(1-11)$ \\
\hline $\begin{array}{l}\text { Nombre d'animaux présentant des } \\
\text { performances faibles (3) } \ldots \ldots \ldots \ldots . . . \\
\text { Numbcr of animals with bad perfor. } \\
\text { mances (3) }\end{array}$ & & & \\
\hline $\begin{array}{l}\text { Prsm:ère semainc } \ldots \ldots \ldots \ldots \ldots \ldots \\
\text { First week }\end{array}$ & $4(4)$ & $3(6)$ & $2(4)$ \\
\hline $\begin{array}{l}\text { Deuxièmz semainc } \ldots \ldots \ldots \ldots \ldots \\
\text { Second week }\end{array}$ & $1(4)$ & $1(2)$ & $0(1)$ \\
\hline $\begin{array}{l}\text { Troisièmz semaine } \ldots \ldots \ldots \ldots \\
\text { Third week }\end{array}$ & $0(5)$ & $0(0)$ & $0(4)$ \\
\hline $\begin{array}{l}\text { Quatrième semaine } \\
\text { Fourth week }\end{array}$ & $1(2)$ & $\mathbf{I}(0)$ & $0(0)$ \\
\hline $\begin{array}{l}\text { Cirquième semaine } \ldots \ldots \ldots \ldots \ldots \\
\text { Fifth week }\end{array}$ & $0(0)$ & $1(1)$ & $0(0)$ \\
\hline
\end{tabular}

(I) Les 5 veaux disparus sont morts dès la première semaine.

The 5 calves missing died during the first week.

(2) Entre parenthèses, valeurs cxtrêmes (j).

Between brackets, extreme values $(d)$.

(3) Le premier chiffre indique le nombre de veaux perdant du poids ou n'en prenant pas et le second (entre parenthèses) le nombre d'animaux de croissance significativement inférieure à celle de la moyenne du lot (moins de $200 \mathrm{~g}$ gain/j).

The first figure is the number of calves with zero or negative liveweight gain and the second (between brackets) is the number of calves with a significantly lower growth rate than that of the others (less than $200 \mathrm{~g}$ gain/d). 


\section{TABLEAU 3}

Evolution des performances zootechniques des veaux au cours de l'expérience (moyenne et écart-type de la moyenne).

Variations of animal performance (mean and standard error of the mean).

\begin{tabular}{|c|c|c|c|}
\hline $\begin{array}{l}\text { Aliment d'allaitement } \\
\text { Milk replacer }\end{array}$ & $\begin{array}{l}\text { «Suif } » \\
\text { «Tallow» }\end{array}$ & $\begin{array}{c}\text { « Suif-coprah-TC6 } » \\
\text { «allow-coconut } \\
\text { oil-TC6 }\end{array}$ & $\begin{array}{l}\text { « Suif-coprah-TC8 } \\
\text { «Tallow-coconut } \\
\text { oil-TC8 }\end{array}$ \\
\hline $\begin{array}{l}\text { Gain de poids des veaux }(\mathrm{g} / \mathrm{j})(1) \\
\text { Liveweight gain of the calves } \\
(g / d)(1)\end{array}$ & & & \\
\hline $\begin{array}{l}\text { Première semaine } \ldots \ldots \ldots \ldots \ldots \\
\text { First week }\end{array}$ & $225 \pm 70$ & $292 \pm 44$ & $266 \pm 65$ \\
\hline $\begin{array}{l}\text { Deuxième semaine } \ldots \ldots \ldots \ldots \ldots \\
\text { Second week }\end{array}$ & $401^{a} \pm 46$ & $571^{b} \pm 44$ & $597 \pm 43$ \\
\hline $\begin{array}{l}\text { Troisième semaine } \ldots \ldots \ldots \ldots \ldots \\
\text { Third week }\end{array}$ & $639^{a} \pm 58$ & $827^{b} \pm 44$ & $786^{b} \pm 31$ \\
\hline $\begin{array}{l}\text { Quatrième semaine } \ldots \ldots \ldots \ldots \ldots \\
\text { Fourth week }\end{array}$ & $830^{a} \pm 90$ & $917^{a b} \pm 44$ & $974 b \pm 45$ \\
\hline $\begin{array}{l}\text { Cinquième semaine } \ldots \ldots \ldots \ldots \ldots \\
\text { Fifth week }\end{array}$ & $939 \pm 45$ & $1000 \pm 31$ & $987 \pm 29$ \\
\hline $\begin{array}{l}\text { Matière sèche consommée }(\mathrm{kg} / \mathrm{ani}- \\
\text { mal) } \ldots \ldots \ldots \ldots \ldots \ldots \ldots \ldots \ldots \ldots \ldots \\
\text { Dry matter intake (kg/animal) }\end{array}$ & & & \\
\hline $\begin{array}{l}\text { Première semaine } \ldots \ldots \ldots \ldots \ldots \ldots \\
\text { First week }\end{array}$ & $4,82 \pm 0,04$ & $4,85 \pm 0,05$ & $4,84 \pm 0,05$ \\
\hline $\begin{array}{l}\text { Deuxième semaine } \ldots \ldots \ldots \ldots \ldots \\
\text { Second week }\end{array}$ & $5,81 \pm 0,11$ & $6,15 \pm 0,10$ & $6,05 \pm 0,11$ \\
\hline $\begin{array}{l}\text { Troisième semaine } \ldots \ldots \ldots \ldots \ldots \\
\text { Third week }\end{array}$ & $6,90 \pm 0,12$ & $7,36 \pm 0,09$ & $7,15 \pm 0,10$ \\
\hline $\begin{array}{l}\text { Quatrième semaine } \ldots \ldots \ldots \ldots \ldots \\
\text { Fourth week }\end{array}$ & $7,76 \pm 0,11$ & $8,40 \pm 0,10$ & $8,30 \pm 0,10$ \\
\hline $\begin{array}{l}\text { Cinquième semaine } \ldots \ldots \ldots \ldots \ldots \\
\text { Fifth week }\end{array}$ & $8,46 \pm 0,09$ & $9,18 \pm 0,08$ & $8,93 \pm 0,08$ \\
\hline
\end{tabular}


TABlEAU 3 (suite)

\begin{tabular}{|c|c|c|c|}
\hline $\begin{array}{l}\text { Aliment d'allaitement } \\
\text { Milk replacer }\end{array}$ & $\begin{array}{l}\text { «Tallow» } \\
\text { «Suif } ~\end{array}$ & $\begin{array}{c}\text { «Suif-coprah-TC6 } \\
\text { «Tallow-coconut } \\
\text { oil-TC6 } »\end{array}$ & $\begin{array}{c}\text { « Suif-coprah-TC8 } \\
\text { «Tallow-coconut } \\
\text { oil-TC8 }\end{array}$ \\
\hline $\begin{array}{l}\text { Efficacité alimentaire globale (g gain } \\
\text { de poids vif } / \mathrm{kg} \text { MS ingérée) (2) .. } \\
\text { Overall feed efficiency } \\
\text { (g liveweight gain } / \mathrm{kg} \text { DM intake) (2) }\end{array}$ & & & \\
\hline $\begin{array}{l}\text { Première semaine } \ldots \ldots \ldots \ldots \ldots \ldots \\
\text { First week }\end{array}$ & 326 & 412 & 385 \\
\hline $\begin{array}{l}\text { Deuxième semaine } \ldots \ldots \ldots \ldots \ldots \\
\text { Second week }\end{array}$ & 484 & 650 & 692 \\
\hline $\begin{array}{l}\text { Troisième semaine } \ldots \ldots \ldots \ldots \ldots \\
\text { Third week }\end{array}$ & 649 & 787 & 769 \\
\hline $\begin{array}{l}\text { Quatrième semaine } \ldots \ldots \ldots \ldots \\
\text { Fourth week }\end{array}$ & 748 & 764 & 821 \\
\hline $\begin{array}{l}\text { Cinquième semaine } \ldots \ldots \ldots \ldots \\
\text { Fifth week }\end{array}$ & 777 & 762 & 774 \\
\hline $\begin{array}{l}\text { Pour } 3 \text { semaines d'essai ........... } \\
\text { For } 3 \text { weeks of experiment }\end{array}$ & 506 & 643 & 639 \\
\hline $\begin{array}{l}\text { Pour } 5 \text { semaines d'essai .......... } \\
\text { For } 5 \text { weeks of experiment }\end{array}$ & 601 & 729 & 716 \\
\hline
\end{tabular}

(1) Les veaux étaient âgés de 2 semaines en début d'essai.

The calves were 2 weeks old at the start of the trial. (2) Valeur globale pour l'ensemble du lot, $\mathrm{y}$ compris les veaux qui ont perdu du poids.
Total value for the whole group of calves, including those which lost weight.

$a, b=$ Les valeurs affectées d'exposants sont significativement différentes $(P<0,05)$. Data with different superscripts are significantly different $(P<0.05)$.

la troisième semaine ( 21 et 18 p. 100 d'augmentation respectivement) et n'est observé en quatrième semaine que pour le lot "suif-coprah-TC8 " (10 p. 100 d'augmentation).

Les 3 lots présentent ensuite des efficacités alimentaires très voisines.

Pour l'ensemble des 3 ou des 5 premières semaines d'expérience, l'efficacité alimentaire globale est ainsi accrue de 27 p. 100 ou de 26 p. 100 dans le premier cas et de 21 p. 100 ou de 19 p. 100 dans le second cas avec les laits "suifcoprah-TC6 " ou "suif-coprah-TC8 " respectivement (tabl. 3).

En raison des teneurs en énergie brute des aliments «suif-coprah-TC6 " et « suif-coprah-TC8 " inférieures à celle de l'aliment "suif » (tabl. 1), l'amélioration de l'efficacité d'utilisation de l'énergie brute est encore plus importante que dans 
le cas de la matière sèche au cours des deuxième et troisième semaines de l'expérience.

De plus, pour l'ensemble des animaux qui ont présenté une croissance positive, aussi faible soit-elle, la moyenne des efficacités individuelles cumulées de l'utilisation de l'énergie est significativement accrue tout au long de l'expérience (fig. 1). Les améliorations sont de 28 p. 100 et de 33 p. 100 après 3 semaines ou de 15 p. 100 et de 19 p. 100 après 5 semaines dans le cas des lots « suif-coprah-TC6 » et « suifcoprah-TC8 » respectivement.

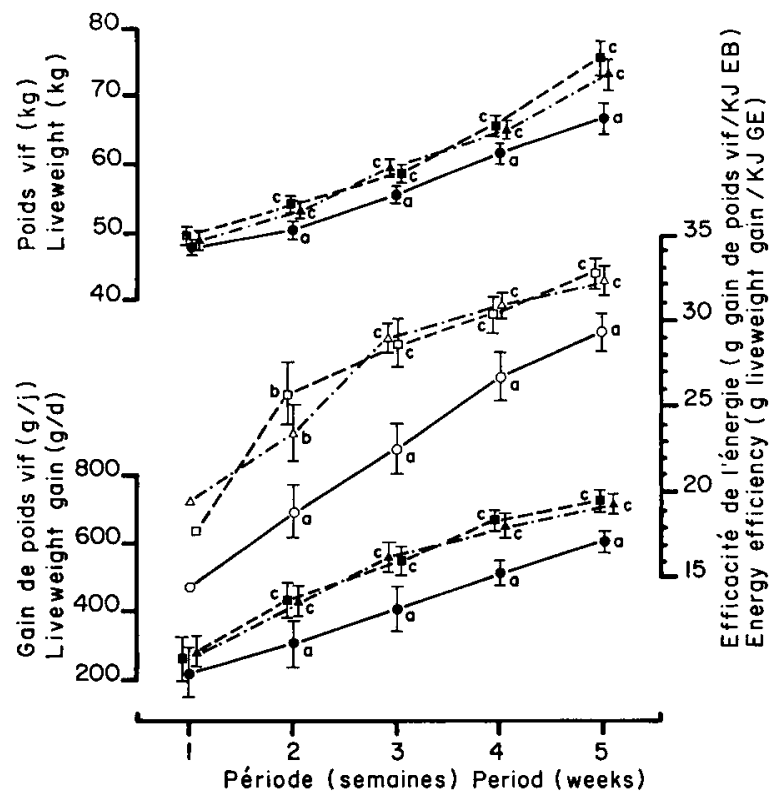

FIG. 1

Performances des veaux utilisés pour la totalité de l'expérience.

Performance of calves during the whole trial.

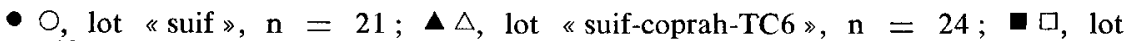
«suif-coprah-TC $8 》, \mathrm{n}=22$. Evolution pondérale (symboles noirs, graphique supérieur), vitesse de croissance depuis le début de l'expérience (symboles noirs, graphique inférieur) et efficacité de l'énergie depuis le début de l'expérience (symboles blancs) : moyenne et écart-type de la moyenne; $a b=$ différence significative au seuil $\mathbf{P}<0,05 ;$ ac $=$ différence significative au seuil $\mathbf{P}<0,025$.

- $\mathrm{O}$, «tallow $»$ group, $n=21 ; \Delta \triangle$, $n$ tallow-coconut oil-TC6» group, $n=24$; - $\square$, tallow-coconut oil-TC8 group, $n=22$. Liveweight variations (black symbols, upper graph), growth rate from the start of the trial (black symbols, lower graph) and energy efficiency from the start of the trial (open symbols): mean and standard error of the mean; $a b=$ level of significance $P<0.05 ; a c=l e v e l$ of significance $P<0.025$. 


\section{*}

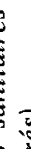

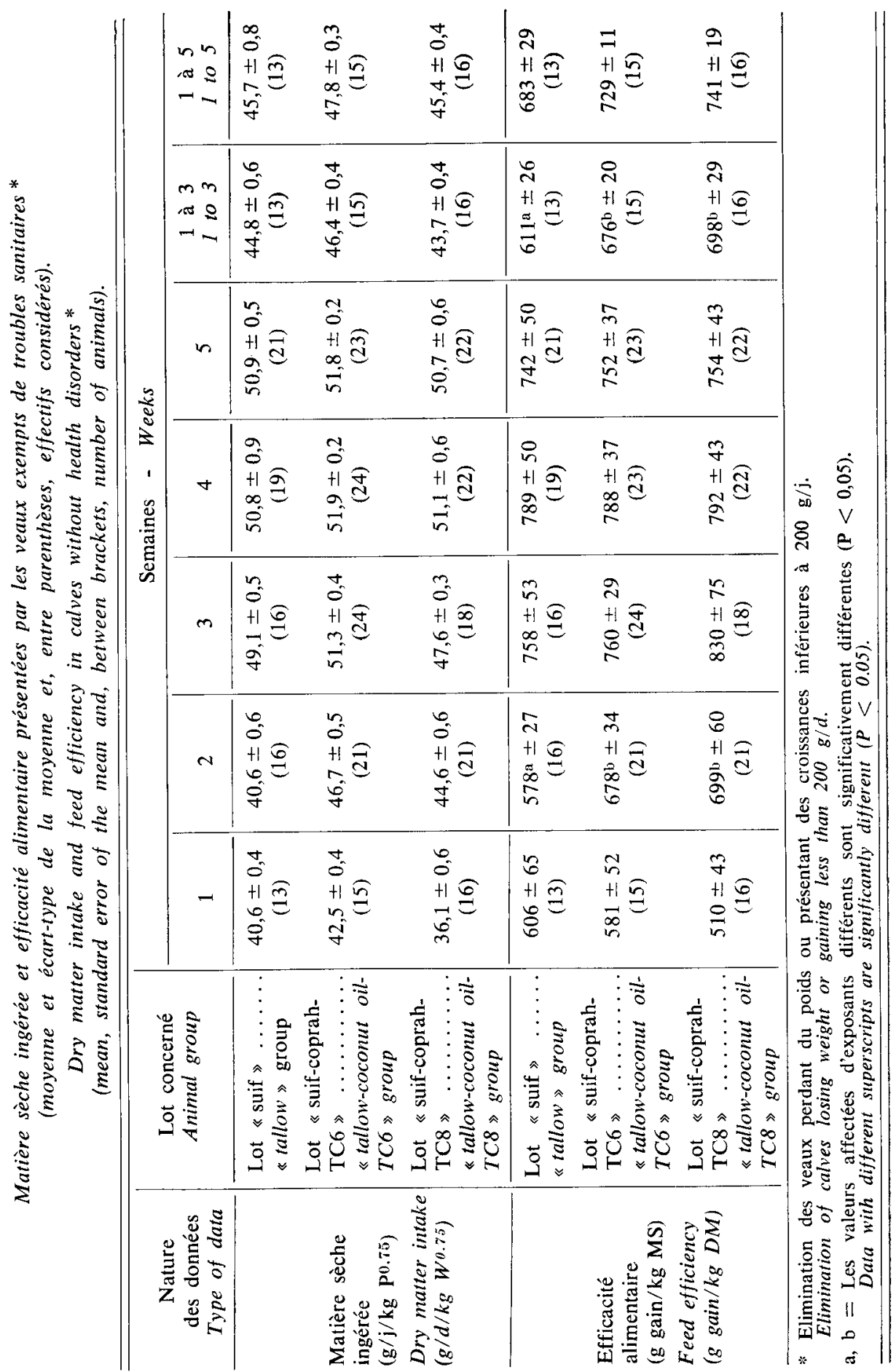


Pour pouvoir comparer dans de meilleures conditions l'utilisation des aliments d'allaitement, les données correspondant aux animaux qui ont eu un gain de poids inférieur à $200 \mathrm{~g}$ par jour ont été éliminées. Les nombres d'animaux retenus pour chaque semaine d'expérience sont indiqués dans le tableau 4. Leur consommation moyenne d'aliment ( $\mathrm{g}$ MS par $\mathrm{kg}$ de poids métabolique) au cours de l'expérience est sensiblement plus élevée $(+5 \mathrm{p}$. 100) pour le lot «suif-coprah-TC6 " que pour les 2 autres lots. Ces différences sont liées à des refus plus fréquents présentés par les veaux des 2 autres lots (lot "suif " en particulier); elles sont partiellement dues, dans le cas du lot «suif-coprah-TC8», à une efficacité alimentaire sensiblement accrue qui aboutit à l'obtention d'animaux plus lourds pour une même quantité d'aliments ingérés.

Pour les animaux retenus, l'efficacité alimentaire est améliorée significativement $(\mathrm{P}<0,05)$ en deuxième semaine (de 17 et 21 p. 100 pour les lots "suif-coprah-TC6 * et « suif-coprah-TC8 » respectivement). Au cours de la troisième semaine, l'amélioration disparaît dans le cas du lot "suif-coprah-TC6" mais elle est encore de 9 p. 100 pour le lot «suif-coprah-TC8 " (N.S.) et elle s'annule par la suite pour les 2 lots. L'efficacité alimentaire est ainsi accrue de 11 p. 100 ou de 17 p. 100 $(\mathrm{P}<0,05)$ pour l'ensemble des trois premières semaines et de $6 \mathrm{p} .100$ ou de 8 p. 100 (N.S.) pour l'ensemble des 5 semaines d'expérience dans le cas des aliments «suif-coprah-TC6 » ou « suif-coprah-TC8 » respectivement.

\section{Discussion}

Les résultats de cette étude permettent de différencier 2 types d'effets bénéfiques de l'ingestion d'acides gras à chaîne moyenne par le très jeune veali : le premier, sur l'état sanitaire, le second sur l'utilisation des aliments d'allaitement pour la croissance.

En effet, au cours de la première semaine d'expérience, l'état sanitaire des veaux des 3 lots était également médiocre; son amélioration plus rapide dans le cas des lots «suif-coprah-TC6 » et «suif-coprah-TC8 " est à l'origine d'une meilleure efficacité globale d'utilisation des aliments par l'ensemble des animaux de ces 2 lots $(+20$ p. 100 , environ, en première et en deuxième semaines, +14 p. 100 environ, au cours des 3 premières semaines); cette amélioration de l'état sanitaire des veaux semble due à un effet favorable des AGCM, car plusieurs de leurs propriétés peuvent l'expliquer :

- en premier lieu, les AGCM sont susceptibles de présenter dans la lumière intestinale une action antimicrobienne efficace (KABARA, VRABLE \& LieKENJIE, 1977);

- de plus, ils peuvent être absorbés à travers l'épithélium intestinal contre un gradient de concentration (ZurIer, Patterson \& Levitan, 1970) ou contre un gradient de pH (BARRY \& SMITH, 1960). Ces composés peuvent également être soumis activement à la $\beta$ oxydation dans les tissus intestinaux lors de leur absorption (Greenberger, Franks \& Isselbacher, 1965) et approvisionner efficacement l'entérocyte en unités acétate susceptibles à leur tour de favoriser l'absorption d'eau et de diminuer la gravité des diarrhées (Demigne et al., 1981). Ainsi, chez l'agneau préruminant, des améliorations spectaculaires des digestibilités de la matière sèche, 
de, l'énergie et de l'azote sont observées lorsqu'on incorpore des AGCM dans des laits offerts à des animaux diarrhéiques (Aurousseau, non publié).

Par ailleurs, nous n'avons pas noté de sensibilité plus grande aux affections pulmonaires chez les veaux recevant des AGCM, contrairement aux observations de Roy et al. (1973 b).

En outre, l'élimination des résultats correspondant aux animaux présentant un mauvais état sanitaire (perte de poids ou gain anormalement faible, inférieur à $200 \mathrm{~g} / \mathrm{j}$ ) suggère qu'un effet bénéfique direct des AGCM sur l'utilisation des aliments d'allaitement pour la croissance s'ajouterait aux effets obtenus grâce à l'amélioration de l'état sanitaire des animaux. L'amélioration supplémentaire de l'efficaciié alimentaire au cours des 3 premières semaines est significative. Elle s'exerce transitoirement, surtout en deuxième semaine $(+20$ p. 100). L'accroissment du CUD a de l'énergie de 3 points dans le cas de l'aliment "suif-coprah-TC6 » et de 2 points dans le cas de l'aliment "suif-coprah-TC8 " (Aurousseau, Vermorel \& Bouvier, 1983, 1984) est insuffisant pour rendre totalement compte de cette amélioration. En effet, l'efficacité d'utilisation de l'énergie digestible de ces 2 aliments est supérieur de 19 et 25 p. 100 à celle de l'aliment "suif " au cours de la deuxième semaine.

L'effet positif des AGCM sur l'efficacité alimentaire serait donc la conséquence directe des augmentations des quantités de protéines fixées dans les tissus (AurousSEAu, Vermorel \& Bouvier, 1983, 1984). Cependant, il est difficile d'expliquer pourquoi les effets sur la croissance des animaux et sur l'efficacité alimentaire sont du même ordre avec les 2 types de matières grasses alors que les effets sur la fixation des protéines étaient bien supérieurs dans le cas du mélange «suif-coprahTC6 ». Pour des raisons pratiques, il n'a pas été possible d'inclure dans l'expérience la mesure de l'efficacité alimentaire d'un lait dans lequel $1 / 3$ du suif aurait été remplacé par de l'huile de coprah. On peut noter à ce propos une amélioration de 12 p. 100 de l'efficacité alimentaire d'un lait dans lequel la totalité des matières grasses étaient apportées par de l'huile de coprah chez l'agneau (Theriez et al., 1973). En revanche, aucun effet n'a été observé dans le cas de laits comportant entre $1 / 3$ et $1 / 2$ des matières grasses sous forme d'huile de coprah, aussi bien chez l'agneau (observations non publiées) que chez le veau (Toullec, communication personnelle ; Roy et al., 1973 b). Nos résultats confirment les observations de NamiotKEVICZ (1973) relatives aux effets favorables des acides gras de longueur de chaîne inférieure à 10 carbones (obtenus ici en présence d'acides laurique et myristique) sur la croissance du veau préruminant. Ils permettent de préciser que les effets favorables des AGCM sur l'efficacité d'utilisation du lait pour la croissance sunt limités à une courte période du début de la vie du veau préruminant comme l'avait observé préalablement VEEN (1970) avec de l'huile de palmiste. Nos résultats sont également en accord avec ceux qui ont été obtenus chez l'agneau préruminant (Aurousseau, 1984, données non publiées) et chez l'enfant nouveau-né (GraceY, Burke \& ANDERson, 1970; Guy-Grand \& Bour, 1970; TANTIBHEdyangkul \& HaShIm, 1971). En revanche, ils s'opposent à ceux rapportés chez le porcelet (NewPort, Storry \& Tuckley, 1979) ou à ceux de OKamoto et al. (1982) chez l'enfant prématuré. De la même façon, des résultats variables ont été relevés chez le rat : effets favorables sur la fixation de protéines (Vermorel \& Aurousseau, 1970 ; Chenat, Aurousseau \& Vermorel, 1976), réduction des quantités de lipides fixés (Aurousseau, de Groot \& Vermorel, 1970 ; Aurousseau, 1972) ou absence complète d'effets (Aurousseau et al., 1978 ; Demarne et al., 1978). 
D’une manière générale, des effets positifs sur la croissance ont été obtenus chez les sujets les plus jeunes (bébé veau et agneau au cours des premières semaines de vie, rat prépubère) mais n'ont pas été observés, dans le cas des animaux présentant une aptitude plus grande à la lipogénèse (porcelet, rat pubère).

\section{Conclusion}

Les résultats publiés dans cette expérimentation en lots confirment l'intérêt potentiel de l'utilisation des acides gras à chaîne moyenne dans l'alimentation des jeunes animaux préruminants. Leur effet favorable sur l'état sanitaire des veaux a permis l'obtention de vitesses de croissance homogènes et s'est traduit globalement par une amélioration de 14 p. 100 environ de l'efficacité d'utilisation des aliments d'allaitement par l'ensemble des lots expérimentaux au cours des 3 premières semaines d'expéricnce. Leurs effets positifs sur la fixation de protéines dans l'organisme permet une amélioration supplémentaire de 20 p. 100 environ de la vitesse de croissance des veaux et de l'efficacité alimentaire au cours de la deuxième semaine d'expérience ou de 12 p. 100 environ au cours des 3 premières semaines. Ces propriétés des AGCM s'ajoutent à leur excellente digestibilité et à leurs effets positifs sur l'utilisation digestive des autres constituants des aliments d'allaitement mis en évidence antérieurement chez les animaux les plus jeunes. Or, le jeune veau préruminant présente au cours des premiers jours après la naissance des capacités d'ingestion et de digestion limitées. De plus, dès l'âge de 8 jours, cet animal est exposé dans les circuits de commercialisation à des agressions multiples qui peuvent favoriser la contamination par des agents pathogènes, déclencher des diarrhées et affaiblir leur organisme (rassemblement de nombreux animaux d'origines multiples, transport sur de longues distances, chocs climatiques et alimentaires). L'ensemble des propriétés des AGCM est donc bien adapté à la résolution des problèmes spécifiques posés par l'organisation de la production du veau de boucherie.

\section{Summary}

Influence of substituting part of the milk replacer tallow by tricaproin or tricaprylin combined with coconut oil on the growth of young preruminant calves

Growth and feed efficiency were compared in 72 calves divided into 3 groups and offered either a conventional «tallow» milk replacer $(23.4$ p. 100 crude protein, 21 p. 100 lipid (group 1) or a «tallow-coconut oil-TC6» milk (group 2) or a «tallowcoconut oil-TC $\$$ milk (group 3 ) in both of which $2 / 3$ of the tallow werc replaced by equal amounts of coconut oil and tricaproin or by equal amounts of coconut oil and tricaprylin, respectively (table 1).

Health status of the calves was bad, so that 5 of them died in the first week, leaving 21, 24 and 22 calves respectively in each of the 3 groups. Incidence of scouring or poor growth and feed efficiency were more severe in group 1 (table 2).

The mean rate of growth of the calves $(+40$ p. 100) as well as dry matter efficiency $(+26$ p. 100) were higher in groups 2 and 3 during the first 3 weeks of the trial (table 3). The increase in energy efficiency was even larger $:+30$ p. 100 after 3 weeks. It was still significant after 5 weeks (fig. 1$):+17$ p. $100(\mathrm{P}<0.05)$. 
After removing the data obtained in poorly growing animals in order to eliminate the effect of health status, groups 2 and 3 still showed higher rate of growth and feed efficiency $(+14$ p. $100, P<0.05$ ) during the first 3 weeks of the trial (table 4$)$. The effects of medium chain fatty acid intake on feed efficiency as related to an effect on health status of the animals or to a direct effect on efficiency of tissue deposition are discussed.

Key words : preruminant calf, caprylic acid, caproic acid, feed efficiency.

Reçu en septembre 1983.

Accepté en décenbre 1983.

\section{Références bibliographiques}

Aurousseau B., 1972. Utilisation énergétique des acides caprylique, laurique et myristique par le rat en croissance. Influence des restrictions alimentaires. Ann. Biol. Anim. Biochem. Biophys., 12, 617-630.

Aurousseau B., De Groot L., Vermorel M., 1970. Etude comparée de l'utilisation énergétique de régimes riches en acide caprylique ou en acides gras insaturés. Ann. Biol. Anim. Biochim. Biophys., 10, 703-706.

Aurousseau B., Perez J.M., Bouvier J.C., Vermorel M., 1978. Influence combinée de l'ingestion de tricaproine et du mode de distribution des repas sur l'utilisation de l'énergie et de l'azote par le rat en croissance. Ann. Biol. Anim. Biochim. Biophys., 18, 1107-1115.

Aurousseau B., Vermorel M., Bouvier J.C., 1983. Influence du remplacement d'une partie du suif d'un aliment d'allaitement par de la tricaproine ou de l'huile de coprah sur l'utilisation de l'énergie et de l'azote par le veau préruminant; influence du niveau d'alimentation antérieur. Reprod. Nutr. Dévelop., 23, 587-598.

Aurousseau B., Vermorel M., Bouvier J.C., 1984. Influence du remplacement d'une partie du suif d'un aliment d'allaitement par de la tricapryline ou de l'huile de coprah sur l'utilisation de l'énergie et de l'azote par le veau préruminant. Reprod. Nutr. Dévelop., 24, 265-279.

Barry R.J.C., Smith D.H., 1960. Transfer of short chain fatty acid by the intestine. J. Physiol., 152, 48-66.

BENNETT S., 1964. Intestinal absorptive capacity and site of absorption of fat under steady state conditions in the unanaesthetised rat. Quart. J. Expl. Physiol., 49, 210-216.

Chenat M.C., Aurousseau B., Vermorel M., 1976. Influence de l'acide caproique sur l'utilisation de l'énergie et de l'azote ingérés par le rat en croissance en fonction de l'âge des animaux et de la tencur en azote des régimes. Ann. Biol. Anim. Biochim. Biophys., 16, 603-622.

De Schothorst, 1966. Proeven aan de afdeling rundvee. Melkvee 67-71 in "Stichting Institut» voor moderne veevoding «De Schothorst». Verlag van de voederproeven in 1966. Waarin opgenomen enkele medelingen van de Stichting voor Onderzoek van Pluimvec en Varkens (Mastrigt en Verhoeven, Arnhem, Pays-Bas).

Demarne Y., Epo N., Flanzy J., Lecourtier M.J., 1978. Comparison of long term lipogenic effects of two different medium-chain triglycerides (TC8-TC12) in the growing rat. Arch. Int. Physiol. Biochem., 86, 25-35.

Demigne C., Remesy C., Chartier F., Lefaivre J., 1981. Effect of acetate or chloride anions on intestinal absorption of water and solutes in the calf. Am. J. Vet. Res., 42, 1356-1359.

Edwards-Webb J.D., Thompson S.Y., 1977. Studies on lipid digestion in the preruminant calf. 2. A comparison of the products of lipolysis of milk fat by salivary and pancreatic lipases in vitro. Br. J. Nutr., 40, 125-131.

Edwards-Webb J.D., Thompson S.Y.., 1978. Studies on lipid digestion in the preruminant calf. 3. The action of salivary lipose on milk fat in the abomasum. Br.J. Nutr., 40, 125-131.

Entressangles B., Pasero L., Savary P., Sarda X., Desnuelle P., 1961. Influence de la nature des chaînes grasses sur la vitesse de leur hydrolyse par la lipase pancréatique. Bull. Soc. Chim. Biol., 43, 581-591. 
Gracey M., Burke V., Anderson C.M., 1970. Medium chain triglycerides in pediatric practice. Arch. Dis. Child., 45, 445-452.

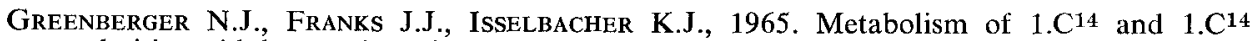
palmitic acid by rat intestinal slices. Proc. Soc. Exp. Biol. Med., 120, 468-471.

GuY-Grand B., Bour M., 1970. Intérêt des triglycérides à chaîne moyenne en diététique clinique. Bull. Acad. Nat. Med., 154, 11-12.

ISSELBACHER K.J., 1968. Mechanisms of absorption of long and medium chain triglycerides. In : J.R. Senior, «Medium chaîn triglycerides», 21, 34. Univ. Pensylvania press.

Kabara J.J., Vrable R., Liekenje M.S.F., 1977. Antimicrobial lipids : natural and synthetic fatty acids and monoglycerides. Lipids, 12, 753-759.

NamıotKevicz J., 1973. L'utilisation des acides gras synthétiques pour l'engraissement des veaux. Rocz. Nauk. Roln., B 94, 7-16.

Newport M.J., Storry J.E., Tuckley B., 1979. Artificial rearing of pigs. 7. Medium chain triglycerides as a dietary source of energy and their effect on live-weight gain, feed : gain ratio, carcass composition and blood lipids. Br. J. Nutr., 41, 85-93.

NiESAR K.M., 1964. Quälitätsmerkmale und Quälitätsbewertung der Fettkomponente in Milchfutter. Bayer. Landw. Jb., 41, 230-242.

Okamoto E., MutTard C.R., Zucker C.L., Heird W.C., 1982. Use of medium chain triglycerides in feeding the low-birth-weight infant. Am. J. Dis. Children, 136, 428-431.

RAVEN A.M., Robinson K.L., 1958. Studies of the nutrition of the young calf. 1. Comparison of starch lactose and hydrogenated palm oil, with butter fat in milk replacers. Br. J. Nutr., 12, 469-482.

Raven A.M., 1970. Fat in milk replacers for calves. J. Sci. Fd. Agric., 21, 352-359.

Roy J.H.B., Stobo I.J.F., Gaston H.J., 1970. The nutrition of the veal calf. 3. A comparison of liquid skim-milk with a diet of reconstituted spray-dried skim-milk powder containing 20 p. 100 margarine fat. Br. J. Nutr., 24, 459-475.

Roy J.H.B., Stobo I.J.F., Gaston H.J., Shotton S.M., Ganderton P., 1973 a. The nutrition of the veal calf. 5. Comparison of two margarine fats. Anim. Prod., 17, 97-107.

Roy J.H.B., Stobo I.J.F., Gaston H.J., Shotton S.M., Ganderton P., 1973 b. The nutrition of the veal calf. 6. The effect of ultra-high (68 p. 100) fat milk powders added to liquid skim milk and a comparison with spray dried skim milk powder containing, 20 p. 100 margarine fat. Anim. Prod., 17, 109-127.

Theriez M., Molenat G., Daniel M., Aurousseau B., 1973. L'allaitement artificiel des agneaux. III. Influence de la nature des matières grasses incorporées dans l'aliment d'allaitement. Ann. Zootech., 22, 185-197.

Toullec R., Mathieu C., 1969. Utilisation digestive des matières grasses et de leurs principaux acides gras par le veau préruminant à l'engrais. Influence sur la composition corporelle. Ann. Biol. Anim. Biochim. Biophys., 9, 139-160.

Toullec R., Thivend P., Vermorel M., Gueguen L., 1978. Veau préruminant. In : Alimentation des ruminants, Ed. I.N.R.A. Publ., Versailles, 245-295.

Tantibhedyangkul P., Hashim S.A., 1971. Clinical and physiological aspect of mediumchain triglycerides : allevation of steatorrhea in premature infants. Bull. N.Y. Acad. Med., 46, 17.

VeEN W.A.G., 1970. Animal and vegetable fats in milk replacers for veal calves. 1. Digestibility coefficients of fats and nitrogen retention. Z. Tierphysiol. Tierernähr. Futtermittelk, 26, 193-202.

Vermorel M., Aurousseau B., 1970. Utilisation énergétique des acides acétique et caprylique par le rat en croissance. In : Schurch A., Wenk C., Energy metabolism of farm animals, Proc 5th Symposium Vitznau, Switzerland, EAAP, 185-188.

Zurier R.B., Patterson J.F., Levitan B., 1970. Relationship between net water absorption and hexanoic acid absorption from the intact human jejunum. Am. J. Digest. Dis., 13, 109-112. 